\title{
COUPP, a Heavy-Liquid Bubble Chamber for WIMP Detection: First Tests in the MINOS Near-Detector Gallery
}

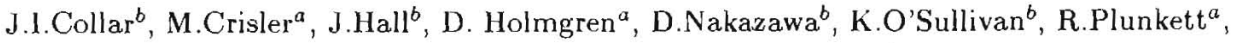 \\ E.Ramberg ${ }^{a}$, A.Raskin $^{b}$, A.Sonnenschein ${ }^{b}$ \\ ${ }^{a}$ Fermi National Accelerator Laboratory, Batavia, IL, USA \\ ${ }^{b}$ Kavli Institute for Cosmological Physics and Enrico Fermi Institute, University of Chicago, IL, USA
}

The Low-Background Detector Development group at the Enrico Fermi Institute (EFI) is presently investigating the application of superheated liquids to WIMP detection. The group has recently demonstrated [1] that it may be possible to keep bulk volumes of heavy refrigerants in a radiation-sensitive metastable state for long enough to perform rare-event searches. For certain choices of operating pressure and temperature the vaporization of the liquid can be produced exclusively by particles having a high stopping power (e.g., nuclear recoils like those expected from Weakly Interacting Massive Particles -WIMPs- or neutrons), making the detector insensitive to minimum ionizing backgrounds. The devices are operated at near room temperature and the industrial refrigerants used are inexpensive, non-flammable and non-toxic, with a chemical composition that maximizes sensitivity to neutralino interactions through both the spin-dependent and independent channels [2]. For these reasons the technique seems to be ideally fitted for the goal of building tonne or even multi-tonne WIMP detectors, devices able to prove most of the supersymmetric phase space where the neutralino dark matter may abide.

Several techniques have been identified and exploited to maximize the stability of small bubble chamber prototypes containing $\mathrm{CF}_{3} \mathrm{Br}, \mathrm{CF}_{3} I$ and $\mathrm{C}_{3} F_{8}$. Namely, avoidance of contact with rough metallic surfaces, use of an immiscible liquid lid above the active volume, outgassing of surfaces in the presence of a buffer liquid, surface cleaning techniques and wetting improvement via vapor deposition [3]. Small prototypes $(\sim 40 \mathrm{~g})$ remain superheated for periods of 15 minutes on the average at the shallow $6 \mathrm{~m}$.w.e. depth of the EFI underground laboratory, a nucleation rate compatible with the measured neutron flux and energy spectrum in the site (Fig. 1). The insensitivity (rejection factor) to minimum ionizing particles (MIPs) in operating conditions at which the liquids are nevertheless fully responsive to low energy nuclear recoils has been measured to be $>10^{9}$ (Fig. 1). This guarantees the ability to build much larger prototypes in the tonne or multi-tonne regime essentially without any concern for MIPs.

Calibrations using neutron sources with a well-defined endpoint energy (11 MeV for $\mathrm{Am} / \mathrm{Be}$ and 152 $\mathrm{KeV}$ for ${ }^{88} \mathrm{Y} / \mathrm{Be}$ ) have allowed to test the response of the liquids to nuclear recoils down to $4 \mathrm{keV}$ in the case of $C F_{3} I$ and to establish agreement with theoretical models of this response (Fig. 1). Data points in the figure represent the appearance of the first bubble nucleation upon decompression in the presence of each source (i.e., as the energy threshold for nucleation is reduced), each point corresponding to a compression/decompression cycle. For sufficiently high source intensities and/or slow decompression rates this bubble (and subsequent violent boiling) is the result of a recoil with an energy close to the highest that the source can produce. These maximum recoil energies are indicated in the labels. The lines represent the theoretical expectations for the onset of sensitivity to recoils of these energies using the classic Seitz "Hot Spike" model [4]. An excellent agreement with the data is observed by best-fitting the single free parameter 

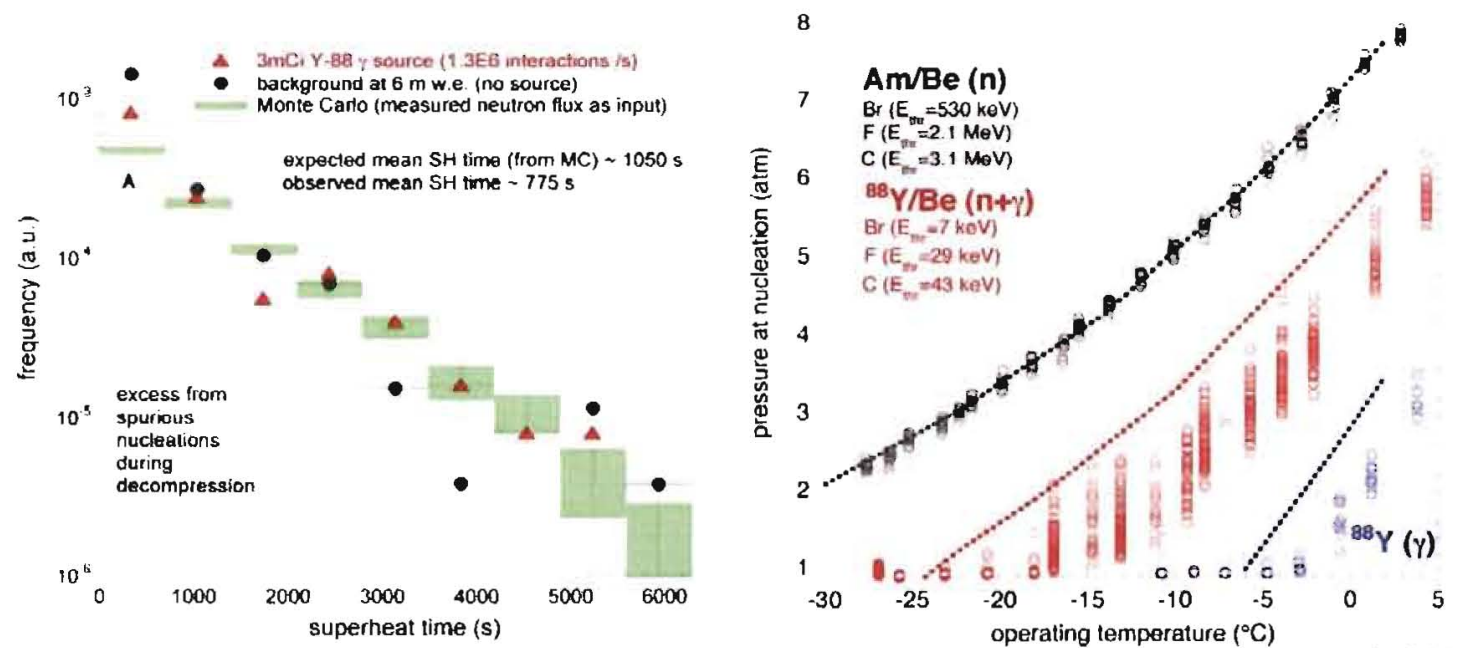

FIG. 1. Left: Distribution of duration of the superheated state in a $12 \mathrm{ml} C F_{3} B r$ bubble chamber at $-10^{\circ} \mathrm{C}$ and atmospheric pressure. Right: Response of the chamber to neutron sources and comparison with theoretical models. Labels indicate the maximum recoil energy that can be produced by the source in each species. Lines indicate the pressure at each temperature below which full sensitivity to this energy is expected according to Seitz's "hot spike" model (the experimental points represent the appearance of the first bubble upon decompression). Complete insensitivity to gamma interactions ( ${ }^{88} Y$ source) in operating conditions that insure full response to low-energy nuclear recoils (e.g., $-10^{\circ} \mathrm{C}$ and $1 \mathrm{~atm}$ ) has been demonstrated in these experiments.

in this model [2] (the best value obtained being compatible with previous bubble chamber studies [4]). The predicted sensitivity for each species not being exactly the same, the lines represent the first one expected to react to the source ( $\mathrm{Br}$ and $\mathrm{F}$, closely matched, in this case). Further calibrations are planned to test the contribution of each individual component to the response of the chamber. The photonuclear ${ }^{88} Y / B e$ source emits a mixed field of $\sim 10^{8}$ high-energy gammas and just $3 \times 10^{3}$ monochromatic neutrons per second, allowing for a dramatic demonstration of total insensitivity to photoelectrons in operating conditions that nevertheless ensure optimal response to WIMP interactions (Fig. 1). A recently procured ${ }^{124} S b / B e$ source will be used to perform calibrations with recoil energies as low as $\sim 1 \mathrm{keV}$, an unprecedented test of a WIMP detector.

The construction of a 1-liter active volume bubble chamber is well advanced (Fig. 2). The purpose of this prototype and experiment (the Chicago Observatory for Underground Particle Physics, COUPP) is to study the ultimate limits to the stability of the superheated liquid in a deeper location, with much reduced neutron backgrounds. However a device of this mass can already be an extremely competitive WIMP detector, given the optimal choice of target nuclei and intrinsic insensitivity to most backgrounds (Fig. 3). Several aspects in the design of this prototype, including safety issues, have been studied by the Fermilab group.

While it is our ultimate goal to deploy a large bubble chamber based dark matter search in the Soudan Mine, we feel that there would be considerable logistical benefits if the initial commissioning and testing of this prototype device take place in the unused "third muon alcove" in the MINOS near detector gallery. Siting the initial commissioning work at Fermilab would allow ready access to the detector for both FNAL and EFI groups, a critical need during the first few months of operation, until the behavior of the detector 
at this depth is completely understood and operation is fully automated. The detector could be ready for underground deployment as early as late summer of 2004. In this FNAL site the experiment would profit from $\sim 300 \mathrm{ft}$ of rock overburden: a preliminary estimate of the cosmic-ray associated backgrounds at this depth reveals that the nucleation rate assumption used to generate the excellent prospects of Fig. 3 could in principle be met there. In the presence of $30 \mathrm{~cm}$ of polyethylene shielding, (cosmic) muon-induced energetic neutrons producing a few nucleations per $\mathrm{kg} / \mathrm{d}$ ay are expected to be dominant, with only a small additional component from beam-related backgrounds (further study of this pulsed component is in progress). The target liquids mentioned above are non-toxic and non-flammable, being as a matter of fact commonly used as fire-extinguishers, and in the case of $C F_{3} l$ and $C_{3} F_{8}$, are not ozone depleting substances. The recompression vessel is ASME code stamped for 600 psi, having undergone hydrostatic tests at up to 800 psi and with a planned operating pressure never surpassing $200 \mathrm{psi}$, but nevertheless features a redundant relief valve(s) system. Extensive discussions with FNAL engineers have resulted in a design meant to assure safety even in catastrophic scenarios such as a fire in the vicinity of the vessel. A containment pan for any eventual small leaks of the non-toxic recompression fluid is part of the design. No high voltages are used for chamber operation, with the exception of the eventual addition of an active muon veto shielding. After a few months of preliminary tests at FNAL the chamber would be transported to a final emplacement in the Soudan mine (MN).

Extensive MCNP-Polimi [5] Monte Carlo simulations of the response to a typical underground neutron flux indicate that large enough bubble chambers (few hundred liters) would have ideal features as WIMP detectors. For instance, a sizeable inner fiducial volume would be shielded against events produced by dreaded "punch-through" neutrons able to penetrate any reasonable thickness of neutron moderator [1]. These represent the ultimate challenge for next-generation WIMP detectors. Their interactions would nevertheless be revealed in these chambers by multi-bubble events which WIMPs cannot produce. To this unique feature one can add the ability to easily exchange liquids from those containing fiuorine as the heaviest atom to those containing iodine or bromine instead: for targets like these the expected WIMP and neutron induced bubble-nucleation rates can be radically different [1], a signature that could be exploited for WIMP discovery. The prototype under construction will also serve the purpose of studying the feasibility of building much larger chambers. New challenges will certainly arise during its operation, besides those already envisioned (for instance, Radon emanation from metallic parts in the inner vessel can give rise to a recoiling-daughter background, even if the prospects based on BOREXINO measurements are reassuring). An active FNAL participation would be enormously beneficial for the future of the project, in view of the existing FNAL expertise in large bubble chamber construction and experience in WIMP searches. 

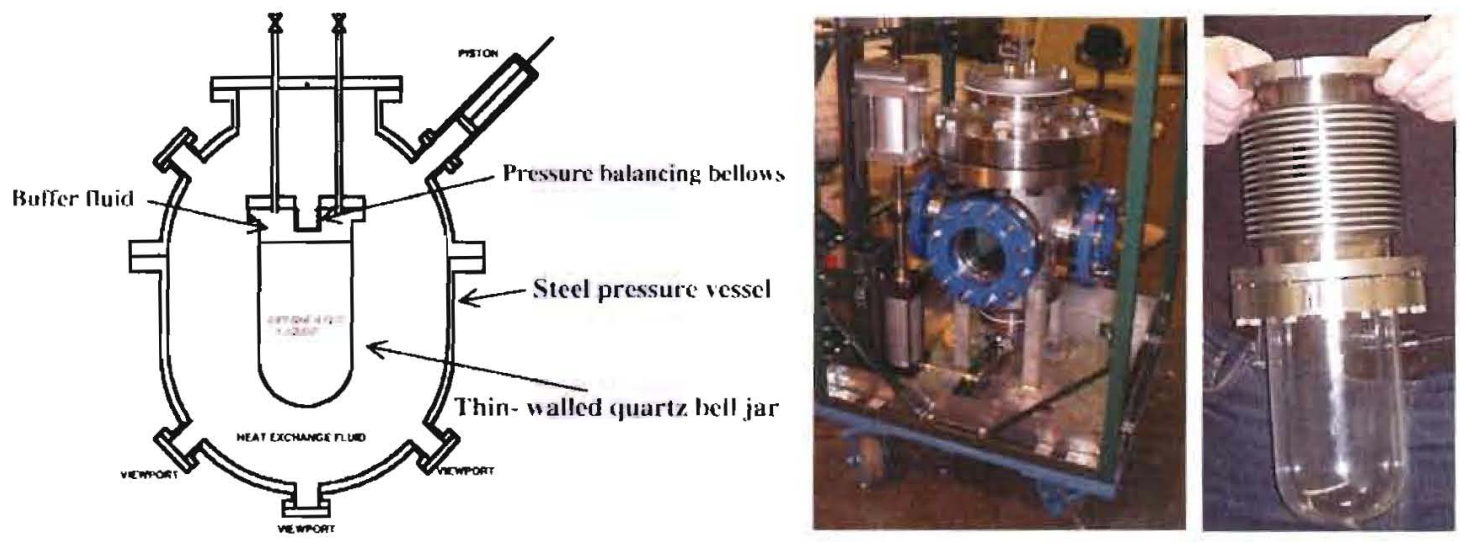

FIG. 2. Left: Conceptual design of the $2 \mathrm{~kg} \mathrm{CF} I$ chamber to be used in the preliminary phase of COUPP. Center: the recompression vessel in this prototype, under construction. Right: inner quartz vial and pressure-compensation bellows for the same.
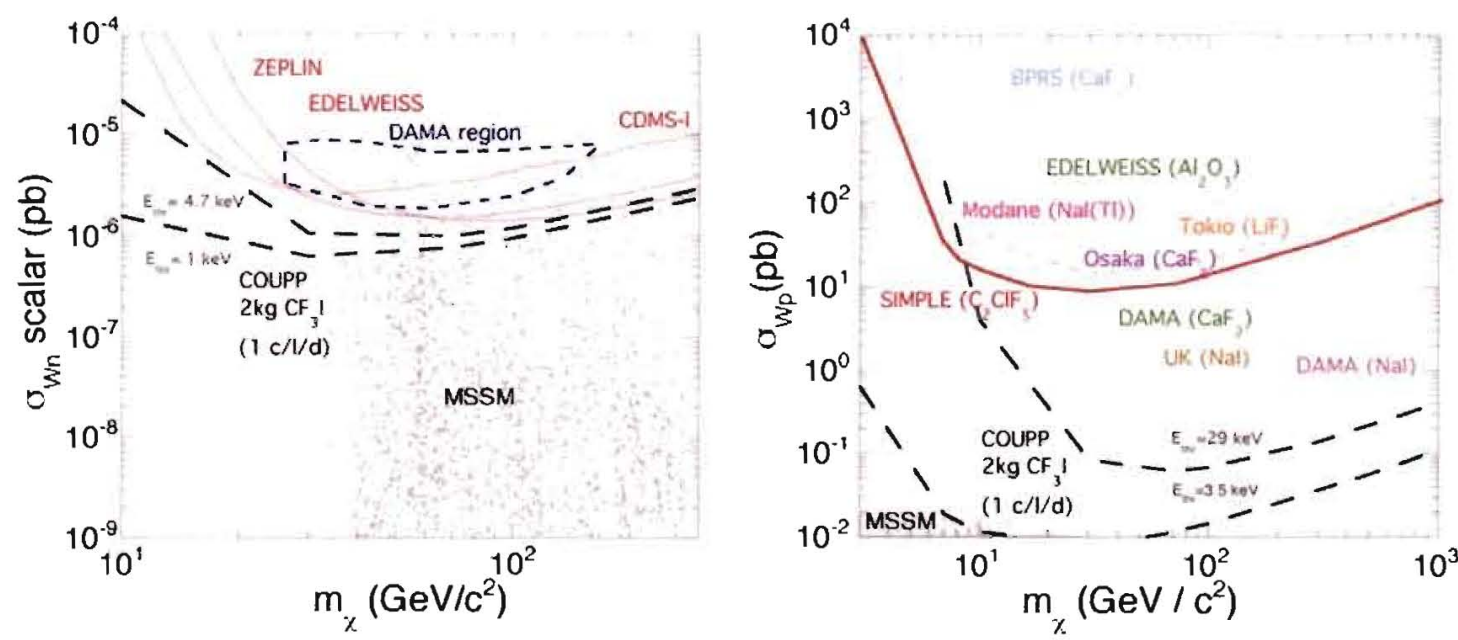

FIG. 3. Sensitivity limits in the spin-independent (left) and spin-dependent (right) neutralino parameter space achievable with COUPP, compared with other experiments. The neutron background rate used for these estimates is representative of what can be expected in the Minos near detector gallery and, according to Monte carlo calculations, a factor of $\sim 100$ too conservative for the Soudan depth. The limits are plotted for two different energy thresholds, one already demonstrated with the $\mathrm{Y}-88 / \mathrm{Be}$ neutron source calibrations and a second one (the best that can be expected before gamma background rejection is lost) soon to be tested with a Sb-124/Be neutron source. NOTE: the recently released CDMS limits surpass those in the figure by a factor $3-4$. 


\section{REFERENCES}

[1] L. Bond et al., Bubble Chambers with Sensitivity to WIMPs, Proc. Topics in Astroparticle and Underground Physics (TAUP 03) University of Washington, Seattle, Washington, 2003. Nucl. Phys. B (Proc. Suppl.) in press.

http://cfcp.uchicago.edu/ $\sim \operatorname{collar} /$ TAUP. pdf http://cfcp.uchicago.edu/ collar/BCposter.pdf

[2] J.I. Collar et al., Phys. Rev. Lett. 85, 3083 (2000) (astro-ph/0001511); J.I. Collar et al., New J. Phys. 2, 14.1 (2000) (http://ww.njp.org).

[3] M.A. Grolmes and H.K. Fauske, Proc. 5th Intl. Heat Transfer Conf., Tokyo 1974; M.G. Buivid and M.V. Sussman, Nature 275 (1978) 203; P. Reinke, Exp. Heat Transfer 10 (1997) 133; P. Reinke, Surface Boiling of Superheated Liquid, Ph.D. Thesis no. 11598, Swiss Federal Institute of Technology, Zurich, Switzerland 1996.

[4] M. Harper, PhD Diss. (U. of Maryland, 1991), Nucl. Sci. Eng. 114, 118 (93), Nucl. Instr. Meth. A336, 220 (93); Ch. Peyrou in "Bubble and Spark Chambers", R.P. Shutt ed., (Academic Press, NY, 1967); F. Seitz, Phys. Fluids 1, 1 (1958); S.C. Roy et al., Nucl. Instr. and Meth. A255, 199 (1987).

[5] S.A. Pozzi et al., Nucl. Instr. Meth. A513 (2003) 550. 
I have added this attachment to the COUPP proposal to provide you with a brief assessment of the scope of the proposal and its impact on Fermilab resources, and to seek your approval and support for the initiation of a Fermilab R\&D effort focused on this promising new detector technology. Over the past week I have been working at the Kavli Institute with Juan Collar and Andrew Sonnenschein on the commissioning of the 1-liter prototype chamber. We successfully condensed CF3I into the chamber Friday night, and on Monday we successfully decompressed. We have already observed stabile superheated operation with an event rate consistent with the expected neutron flux. It is quite exciting.

Our core proposal is to test this new heavy-liquid bubble chamber in the MINOS Near Detector Hall prior to moving it to its ultimate destination in the Soudan Mine. To give a sense of scale, the complete detector is contained in a three-foot square, five-foot high support frame and would be surrounded by about a foot of polyethylene shielding. Based on the very successful preliminary tests, it is likely that we might be ready to move the chamber to Fermilab by early fall. The Kavli Institute will provide the complete chamber, its ancillary equipment, and the polyethylene neutron shield. The Fermilab contributions would include engineering review/ safety analysis, engineering support for the lifting, handling and transport of the device, and coordination of the access to the MINOS hall. I would estimate that a few person-months of engineering support and one or two person-months of drafting support would be sufficient for this test.

Of course a successful test will lead to a more ambitious proposal. To give a sense of how this might develop, a 1-liter device might be followed by a 10-liter version, then by 100 -liters, etc. The M\&S costs will continue to be small. A system consists of vessels (pressure up to $~ 300 \mathrm{psi}$ ), plumbing, a few temperature and pressure sensors, a circulating heater/chiller, video cameras, and a readout computer. There is very little electronics. I believe that it would be difficult to spend much more than $\$ 100 \mathrm{k}$ on a world-class dark matter detector. The opportunities I see for Fermilab participation basically fall in to the areas of bubble chamber engineering, data acquisition and triggering, and in prototype development and testing. What I would like to do immediately is to set up a small lab where we can begin working with the technology here. This would involve scrounging some equipment, and a modest budget for some specialized lab equipment. I don't imagine that this would exceed $\$ 25 \mathrm{k}$. I would also like to involve some Fermilab engineers in thinking about these devices.

What we seek from the Fermilab Directorate is approval to proceed with our core proposal, and beyond that I would hope to get the your blessing to put together a design and development team and to perhaps spend an initial few thousand of PPD's remaining dollars, with the understanding that the cost of this initial effort might grow to $\$ 25 \mathrm{~K}$ or so and would lead to a follow-on proposal for a larger device. 
Draft Memorandum of Understanding

1) Kavli Institute Scientific Staff:

J. Collar, J. Hall, D. Nakazawa, K. O'Sullivan, A. Raskin, A. Sonnenschein

2) Kavli Institute will provide:

a) 1-liter bubble chamber prototype

b) support stand

c) fluid handling system

d) cameras, DAQ computer

e) fluids (purified water, polyethylene glycol, $\mathrm{CF}_{3} \mathrm{I}$ )

f) polyethylene neutron shield

g) possible scintillation counter veto shield

(I have not asked for a detailed

accounting of the Kavli costs. The equipment already exists, and represents about $\$ 50 \mathrm{k}$ )

3) Fermilab Scientific Staff:

M. Crisler, D. Holmgren, R. Plunkett, E. Ramberg, (D. Bauer at a later date)

4) Fermilab will provide:

a) Engineering/Safety Analysis 2 person-months engineering time

i) Preliminary work has already been done by Rich Schmitt.

ii) final engineering and safety analysis and report

iii) pressure vessel certification

iv) approval of fluids for use in underground lab.

b) Engineering Support for transport/handling

2 person-months eng/design/draft

i) lifting fixture plus analysis, documentation

ii) analysis of transport issues

iii) handling underground (method for pushing "up the hill" to the muon alcove)

c) Access to MINOS hall.

i) Some coordination with MINOS will be required due to occupancy limits.

4) Fermilab R\&D effort:

a) lab infrastructure

i) test vessels $\$ 5 \mathrm{k}$

ii) miscellaneous plumbing, hardware $\$ 5 \mathrm{k}$

iii) cameras and optics $\$ 5 \mathrm{k}$

iv) DAQ \& controls computer and software $\quad \$ 5 \mathrm{k}$

v) circulating heater/chiller unit $\$ 5 \mathrm{k}$

Total Initial R\&D infrastructure costs \$25k (not all needs to be purchased new) 


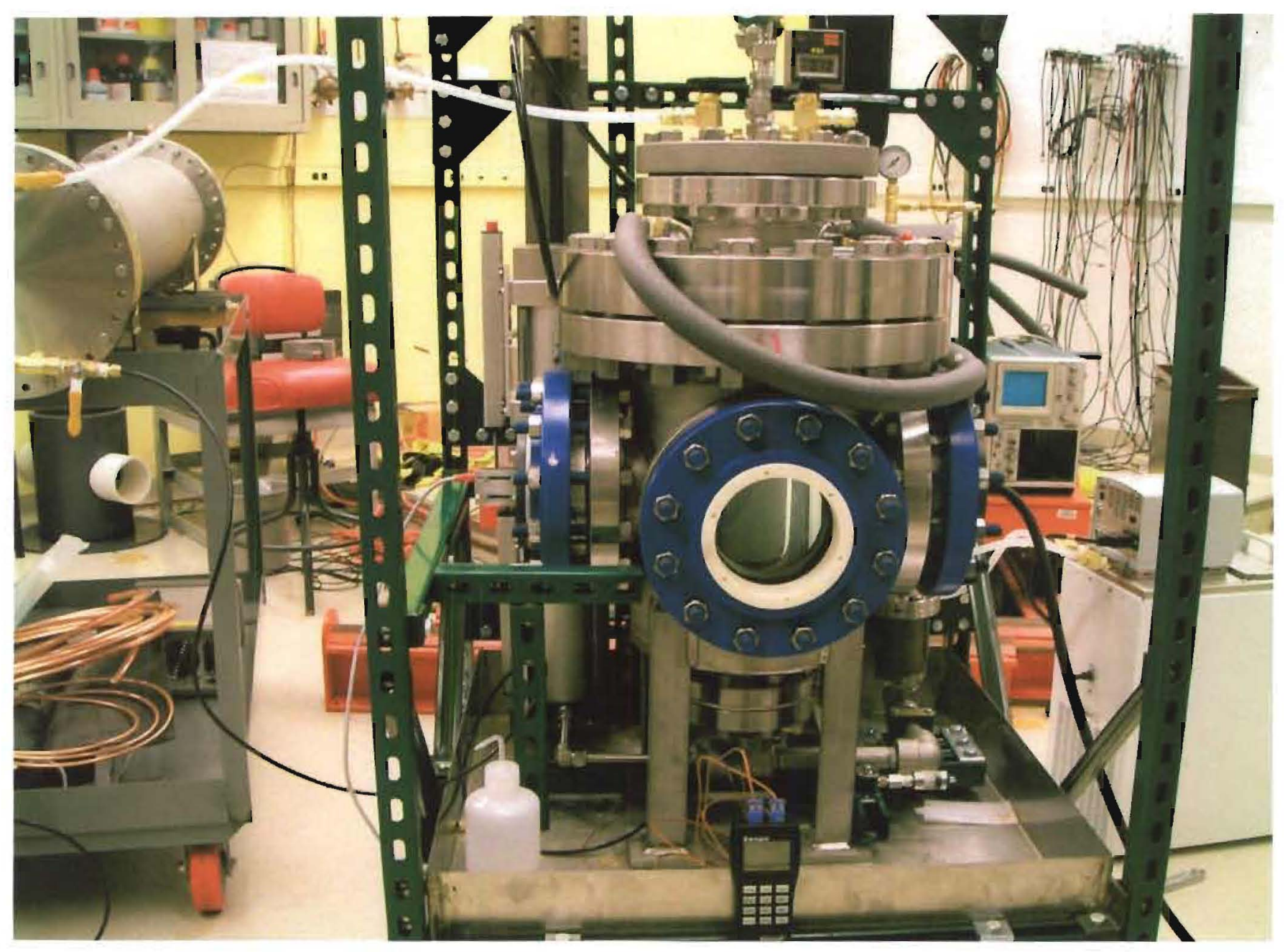

This picture is shows the full outer vessel on its support stand. The inner bubble chamber vessel is visible through the quartz window. 


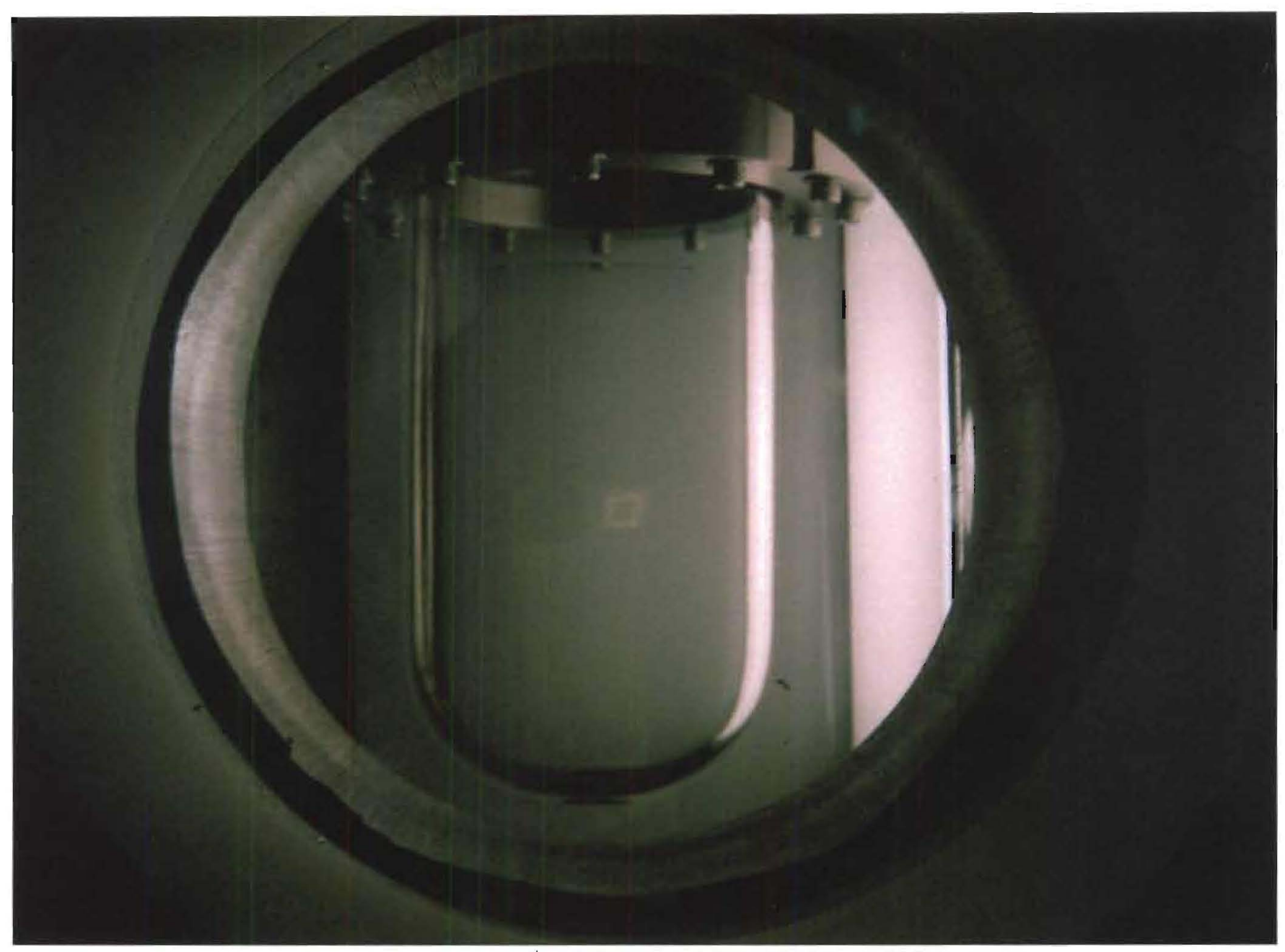

This is a close-up view of the active bubble chamber vessel. Note the meniscus near the top of the quartz vessel indicating the interface between the active $\mathrm{CF}_{3} \mathrm{I}$ fluid (below) and the $\mathrm{H}_{2} \mathrm{O}$ buffer fluid (above). 


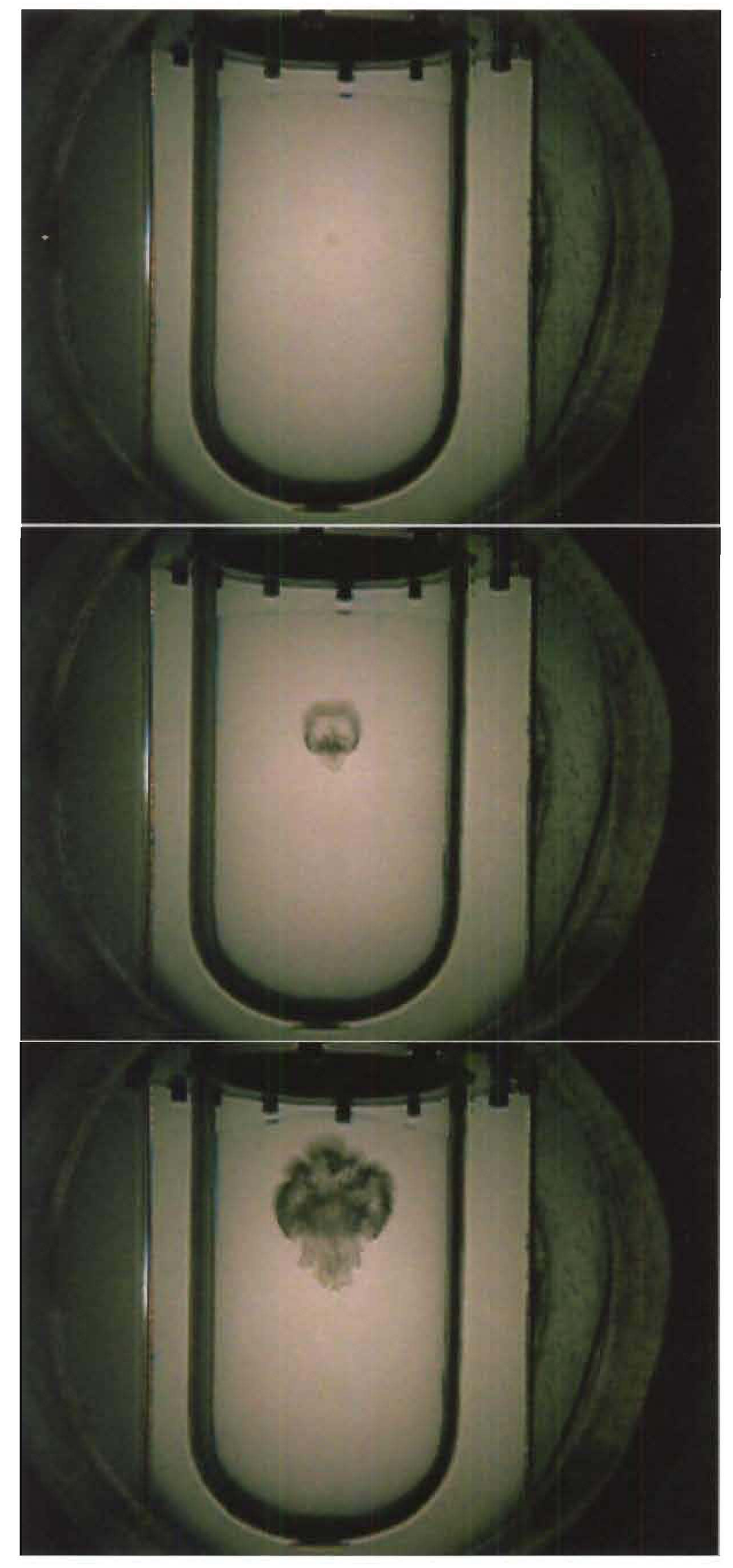

This series of frames shows the formation of a bubble, presumably due to the interaction of an ambient neutron. We've observed a lifetime of order one minute, consistent with the neutron flux in the lab and accounting for the additional shielding effect of the outer polyethylene glycol volume. 